\title{
MicroRNA-29a-3p Downregulation Causes Gab1 Upregulation to Promote Glioma Cell Proliferation
}

\author{
Nai-yuan Shao ${ }^{a}$ Dong-xing Wang ${ }^{b}$ Yin Wang ${ }^{c}$ Ya Lic Zhi-qing Zhang ${ }^{c}$ \\ Qin Jiang ${ }^{d}$ Weifeng Luo $^{b}$ Cong Caob,c,d,e \\ aDepartment of Neurosurgery, the Third Affiliated Hospital of Soochow University, Changzhou, \\ 'Department of Neurology, the Second Affiliated Hospital of Soochow University, Suzhou, 'Institute of \\ Neuroscience, Soochow University, Suzhou, 'The Affiliated Eye Hospital, Nanjing Medical University, \\ Nanjing, eNorth District, The Municipal Hospital of Suzhou, Suzhou, China
}

\section{Key Words}

Glioma $\cdot$ Gab1 $\cdot$ MicroRNA-29a-3p • Cell proliferation

\begin{abstract}
Background/Aims: Glioma causes significant human mortalities annually. Molecularlytargeted therapy is a focus of glioma research. Methods: Grb2-associated binding 1 (Gab1) expression and microRNA-29a-3p ("miR-29a-3p") expression in human glioma cells and tissues were tested by Western blotting assay and qRT-PCR assay. shRNA/siRNA strategy was applied to silence Gab1 in human glioma cells. miR-29a or anti-sense miR-29a construct was transfected to human glioma cells. Cell proliferation was tested by BrdU ELISA assay and cell counting assay. Results: We show that expression of Gab1 was significantly elevated in human glioma tissues and cells, which correlated with downregulation of its putative microRNA: miR29a-3p. In A172 glioma cells and primary human glioma cells, Gab1 shRNA/siRNA inhibited AktErk activation and cell proliferation. Forced-expression of miR-29a-3p downregulated Gab1, inhibiting glioma cell proliferation, whereas miR-29a-3p was in-effective on cell proliferation in Gab1-silenced A172 cells. Furthermore, introduction of a 3'-untranslated region (3'-UTR) mutant Gab1 (UTR-G160A) blocked miR-29a-3p-induced inhibition on Akt signaling and A172 cell proliferation. Conclusions: miR-29a-3p downregulation leads to Gab1 upregulation to promote glioma cell proliferation.

\section{Introduction}

As one common brain tumor, glioma causes significant annual human mortalities [1-3]. Glioblastoma and other high-grade (grade III-IV) gliomas have an extremely poor prognosis [1-3], due to the ineffectiveness of the current treatments, including the surgical

Nai-yuan Shao, Dong-xing Wang, Yin Wang and Ya Li contributed equally to this work.

Qin Jiang M.D. Ph.D., Weifeng Luo and Cong Cao

The Affiliated Eye Hospital, Nanjing Medical University, 138 Han-zhong Road, Nanjing, (China) 210029; Tel. +86-025-86677699, Fax +86-025-86677699

E-Mail Jqin710@vip.sina.com; Lwfwxx@126.com; caocong@suda.edu.cn
\end{abstract}

\section{KARGER}


glioma resection, postoperative radiation and temozolomide chemotherapy [4-6]. Gliomas are characterized by extensive molecular heterogeneity [1], resulting in dysregulated and hyperactivation of multiple signaling pathways, including phosphatidylinositol 3-kinase (PI3K)-Akt and Erk-MAPK cascades, which positively contribute to glioma tumorigenesis and progression [7-9].

Grb2-associated binding (Gab) proteins are scaffolding/adapter family proteins, which include at least three members, Gab1, Gab2, and Gab3 [10-13]. Gab1 is the most predominant Gab family protein [10-13], and can dock with SH2- and SH3-containing signaling proteins [10-13]. Gab1 associates with p85, the PI3K regulatory subunit, to mediate downstream Akt activation [14, 15], whereas Gab1-SHP2 association is necessary for Erk-MAPK activation $[15,16]$. It has previously been shown that Gab1 expression was elevated in human glioma cells [17]. However, the underlying mechanisms of Gab1 upregulation, as well as the potential functions of Gab1 in glioma cell progression have not been extensively studied.

microRNAs (miRNA), a class of endogenous small non-coding regulatory RNAs, can alter gene expression at both translational and post-transcriptional levels [18, 19]. The 1924 nucleotide single-stranded noncoding RNAs bind to the $3^{\prime}$ untranslated region (UTR) of their targeted genes, causing mRNA decay and/or translational block $[18,19]$. miRNA are often dysregulated in human glioma [20-22]. Through searching multiple miRNA databases we identified a novel putative Gab1-targeting miRNA: microRNA-29a-3p ("miR-29a-3p"). Experimental validation shows that miR-29a-3p downregulation leads to Gab1 upregulation to promote glioma cell proliferation.

\section{Materials and Methods}

\section{Ethics approval}

The protocols of this study were according to the principles of Declaration of Helsinki, and were approved by the Soochow University's Ethics Review Board (ERB) and Internal Review Board (IRB).

\section{Reagents}

All the antibodies utilized in this study were described previously [14, 23-26]. Cell culture reagents were from Gibco (Shanghai, China). Puromycin was purchased from Sigma Chemicals (Shanghai, China).

\section{A172 glioma cell line}

The established human glioma A172 cell line was purchased from the Cell Bank of Shanghai Biological Institution (Shanghai, China). Cells was cultivated as described [26, 27]. Every four months, DNA fingerprinting and profiling were performed to confirm the cell line's origin. Cells were subjected to mycoplasma and microbial contamination examination every month. Population doubling time, colony forming efficiency, and morphology were also examined.

\section{Human glioma tissues}

As previously described [28], a total of thirty-two (32) different glioma patients (16 low-grade and 16 high-grade) at the Affiliated Hospitals of Soochow University (Suzhou, China) and Shanghai Xinchao (Shanghai, China), were enrolled. The preparations and examination of fresh human tissue specimens were also described early $[26,28]$. Written informed-consent was obtained from each participant. The protocols of this study were according to the principles of Declaration of Helsinki, and were approved by the Soochow University's ERB and IRB.

\section{Primary culture of human glioma cells and astrocytes}

Establishment and culture of primary human glioma cells were described in detail in our previous studies [26-28]. In short, fresh human glioma tissues were thoroughly washed (in cold PBS), minced and filtered. Single-cell suspensions were obtained through digestion [26], and primary glioma cells were cultured in the described medium [25]. One primary human glioma cell line was established. The origin and culture of primary human astrocytes were also described in our previous study [26]. 


\section{Cellular Physiology Cell Physiol Biochem 2018;48:450-460

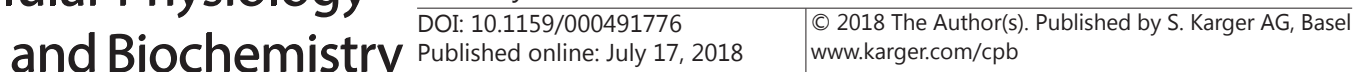

Shao et al.: microRNA-29a Targets Gab1 in Glioma Cells

Quantitative real-time reverse transcriptase polymerase chain reaction ( $q R T-P C R$ ) assay

qRT-PCR was performed using an ABI7600 Prism equipment through the SYBR Green PCR kit. The detailed protocol was described previously [26, 28-32]. Quantification of Gab1 mRNA was through the ${ }^{\Delta \Delta} \mathrm{Ct}$ method [33]. Gab1 mRNA primers were described in previous study [34]. GAPDH was tested as the internal reference gene [26]. The detection of mature hsa-miR-29a/b/c-3p and hsa-miR-29a-5p was examined by the TaqMan microRNA assay using the primers described [35-38]. For each preparation, ten (10) ng of RNA was reverse-transcribed using the TaqMan MicroRNA Reverse Transcription kit (Applied Biosystems, Shanghai, China).

\section{Forced-expression of miR-29a and anti-sense miR-29a ("antagomiR-29a")}

The pre-miR-29a, obtained from Applied Biosystems, was sub-cloned into the HpaI and XhoI sites of pSuper-puro-GFP $[29,39]$ to generate the miR-29a expression vector. The vector was then co-transfected with the packaging plasmids VSVG and Hit-60 [14] via Lipofectamine 2000 to construct the viral particles in HEK293T cells. The viral particles were added to cultured glioma cells. Cells were then subjected to puromycin $(1.0 \mu \mathrm{g} / \mathrm{mL})$ selection for three passages. Control cells were constructed with non-sense scramble microRNA-control ("miR-C") [28]. For permanent inhibition of miR-29a, vectors with an anti-miR29a sequence [40] was packaged into the virus.

\section{Gab1 mRNA 3'-UTR luciferase assay}

The reporter vector with the 3-UTR of Gab1 carrying a putative miR-29a-3p binding site (Position 159-165) was constructed by Genepharm (Shanghai, China). The complementary oligonucleotides for the selected region were hybridized to form double-stranded DNA and inserted into pmIR-ReporterTM firefly luciferase vector (Genepharm). The construct was further co-transfected with the above miR-29a expression vector to A172 glioma cells. Cells were then lysed and analyzed by the luciferase assay kit (Promega, Shanghai, China). $\beta$-galactosidase luciferase was utilized as an internal control.

\section{Western blotting assay}

The detailed protocol for Western blotting assay has been extensively described in our previous studies $[23,24,26]$. Same set of lysate samples were run in sister gels to examine different proteins. For each lane, the exact amount of protein lysates were loaded. Image J software (NIH) was always employed to quantify the intensity of each band.

\section{Gab1 stable knockdown}

Two non-overlapping GV248 lentiviral shRNAs (“-a/-b”, with non-overlapping sequences) against human Gab1 (NM_002039) were described previously [41]. The detailed protocol for shRNA infection and stable cell selection were described in our previous studies [14, 23-26, 31]. The scramble non-sense lentiviral shRNA ("scr-shRNA") [26] was added to the control cells. Gab1 knockdown in the stable cells was verified by Western blotting assay and/or qRT-PCR assay.

\section{Gab1 siRNA}

In the primary human glioma cells, siRNA method was applied to knockdown Gab1. Gab1 siRNA (sc-35431) and a negative control scramble siRNA were purchased from Santa Cruz Biotech (Shanghai, China). siRNA (200 nM each, 24 hours) transfection was performed via the described Lipofectamine 2000 (Invitrogen) method [42].

\section{Gab1 mutation}

Through in vitro site-directed mutagenesis system, a miRNA-29a-binding 3'-UTR-mutant Gab1 (Gab1UTR-G160A, tagged with Flag) vector was constructed and verified by Genepharm (Shanghai, China). The construct was sub-cloned into the GV248 lentiviral vector, and was added to A172 glioma cells. Stable cells were again selected by puromycin (for 3-4 passages). Expression of the mutant Gab1 (UTR-G160A, tagged with Flag) in stable cells was tested by Western blotting assay.

\section{Cell proliferation assay}

Cell counting assay and BrdU incorporation ELISA assay of cell proliferation were described early in our previous studies $[14,23,24,26,31]$.

\section{KARGER}


Statistical analysis

Statistical analysis was performed as previously described $[26,43]$.

\section{Results}

Gab1 upregulation and microRNA29a-3p downregulation in human glioma tissues and cells

To examine Gab1 upregulation in human gliomas, qRT-PCR was employed to test Gab1mRNA expression. As shown in Fig. 1A, Gab1 mRNA levels were significantly elevated in glioma tumor tissues (" $\mathrm{T}$ "), as compared to the surrounding normal brain tissues ("N"). Notably, Gab1 mRNA upregulation was greater in late-stage gliomas (Grade IIIIV, $n=16$ ) than that in early-stage gliomas (Grade I-II, n=16) (Fig. 1A). Western blot analysis confirmed Gab1 protein upregulation in glioma tumor tissue (Fig. 1B, see quantification), results further confirmed greater Gab1 protein upregulation in latestage gliomas than that in early-stage gliomas (Fig. 1B).

Using computational analysis of microRNA databases (TargetScan v7.1), we identified that the 3-UTR of human Gab1 (at position 159165) (Fig. 1C) is a virtual target of microRNA-29a-3p ("miR-29a-3p"). miR-29a-3p showed highest percentile scores and low Context+ scores against Gab1 (TargetScan v7.1). Testing the expression of miR-29a-3p in human glioma tissues, we found that miR$29 a-3 p$ levels were significantly decreased in both early-stage and late-stage (Fig. 1D) gliomas, compared to the relatively high levels in normal brain tissue (Fig. 1D). Notably, miR29a-3p downregulation was again more significant in late-stage gliomas (Fig. 1D). In contrast, the expression of other miR-29 family members, miR-29b-3p and miR-29c-3p, were unchanged between glioma tissues and normal brain tissues (Fig. 1E). Thus, miR-29a-3p is downregulated in human glioma tissues.

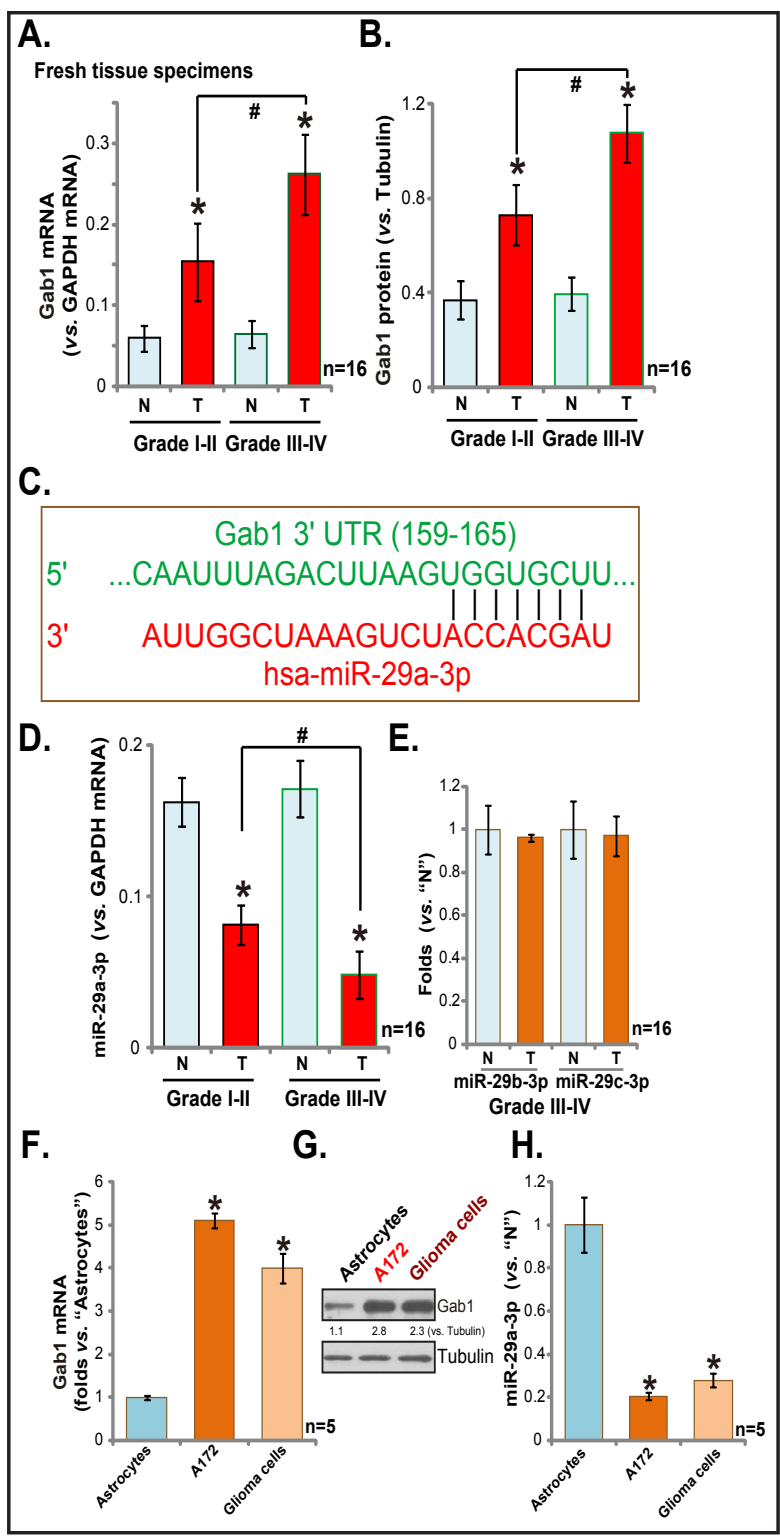

Fig. 1. Gab1 upregulation and microRNA-29a-3p depletion in human glioma tissues and cells. Fresh human glioma tissues ("T") and paired surrounding normal brain tissues ("N") were homogenized and dissolved in the tissue lysis buffer, Gab1 mRNA expression (vs. GAPDH mRNA) (A), microRNA-29a/b/c-3p expression (vs. GAPDH mRNA, D-E) were examined by qRT-PCR assay; Western blotting assay results showed expression of Gab1 in above patients' tissues (results were quantified, B). (C) microRNA-29a-3p ("miR-29a-3p") putatively targets the 3-UTR of human Gab1 (at position 159-165). Expressions of Gab1 mRNA (F), Gab1/Tubulin protein (G) and miR-29a-3p (H) in the listed cells were also shown. "MW" stands for molecular weight (Same for all Fig.). Bars stand for mean \pm SD (Same for all Fig.). * $\mathrm{p}<0.05$ vs. "N" group (A, B and D) or "Astrocytes" (F and H). " p<0.05 (A, B and D). 
The upregulation of Gab1 expression in human glioma cells was correlated with downregulation of miR-29a-3p. As compared to the primary human astrocytes ("Astrocytes") [26, 28], Gab1 (protein and $m R N A$ ) level was again elevated in established A172 glioma cells [26] and primary human glioma cells ("Glioma cells") (Fig. 1F and G) , whereas miR-29a-3p levels were decreased (Fig. $1 \mathrm{H}$ ). These results suggest that miR-29a-3p is a putative antiGab1 miRNA.

\section{Gab1 knockdown inhibits Akt-Erk activation and glioma cell proliferation}

In order to study the function of Gab1 in human glioma cells, two distant lentiviral Gab1 shRNAs with non-overlapping sequences, "shGab1a" and "shGab1b" [41], were applied. Performing qRT-PCR (Fig. 2A) and Western blotting assays (Fig. 2B), we show that both shRNAs potently knocked down Gab1 in A172 glioma cells (Fig. 2A and $\mathrm{B}$ ). Consequently, activation of Akt (pAkt at Thr-308) and Erk (p-Erk1/2 Thr202/Tyr204), two major Gab1 downstream procancerous cascades $[10,12]$, were largely inhibited (Fig. 2B). As expected, expression of miR-29a$3 p$ was not changed with Gab1 knockdown (Fig. 2C). Examining cell proliferation, employing viable cell counting assay and BrdU ELISA assay, we show that Gab1 shRNA knockdown significantly suppressed A172 cell proliferation, as the number of cells (at Day-4, Fig. 2D) and BrdU ELISA OD (Fig. 2E) were both decreased in Gab1-silenced cells.

To study the effect of Gab1 on the primary human glioma cells we utilized the siRNA strategy. The applied Gab1 siRNA dramatically decreased Gab1 mRNA (Fig. 2F) and protein expression (Fig. 2G) in the primary cancer cells. Gab1 silence inhibited Akt and Erk activation (Fig. 2G) and proliferation of the primary cancer cells (Fig. $2 \mathrm{H}$ and I). These in vitro studies in glioma cells demonstrate that Gab1 knockdown inhibits AktErk activation and cancer cell proliferation.

Forced-expression of miR-29a$3 p$ silences Gab1, inhibiting glioma cell proliferation

To study the potential effect of miR-29a on Gab1 expression,

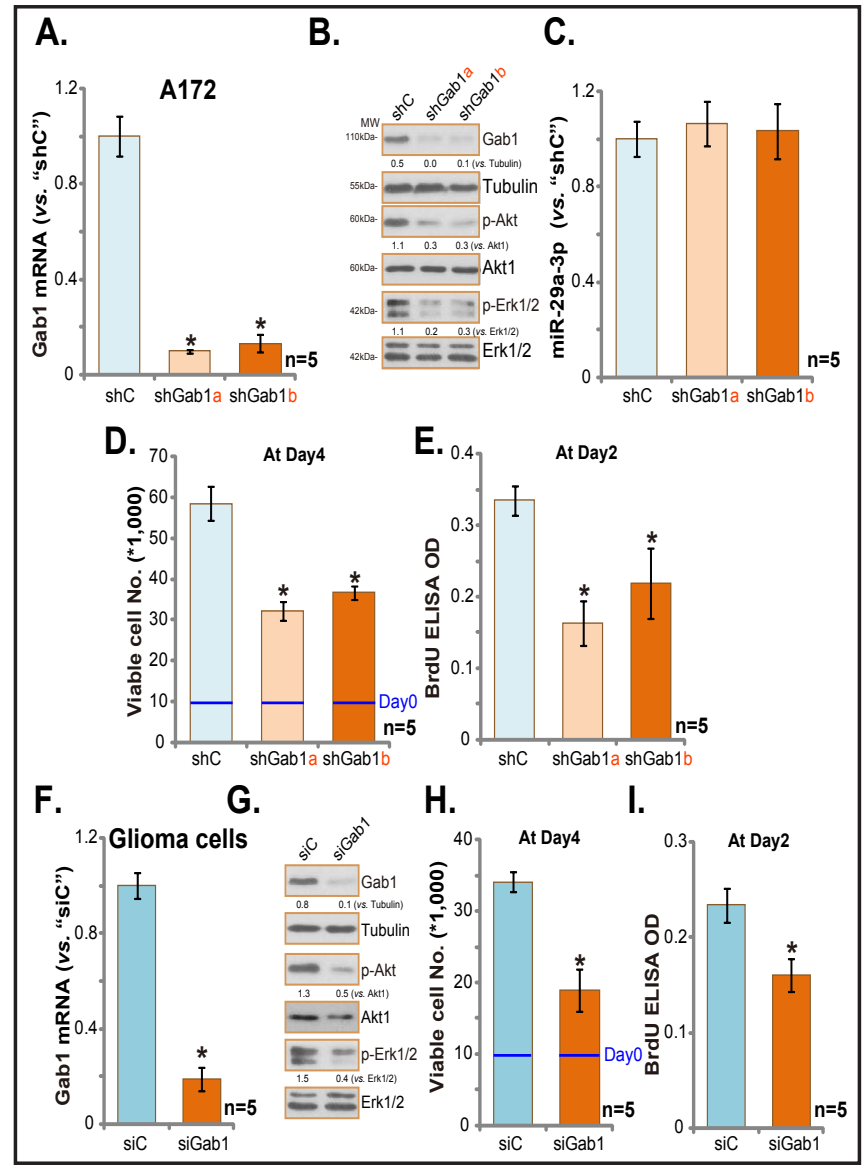

Fig. 2. Gab1 knockdown inhibits Akt-Erk activation and glioma cell proliferation. Stable A172 cells, constitutively expressing Gab1 shRNA ("-a/-b") or scramble control shRNA ("shC"), were subjected to qRT-PCR assay (A, to test Gab1 mRNA) and Western blotting analysis of listed proteins (B); miR-29a-3p expression was also tested (C). Above cells were also subjected to listed proliferation assays (D and E). Expressions of Gab1 mRNA (F) and listed proteins (G) in primary human glioma cells ("Glioma cells"), transfected with 200 nM of Gab1 siRNA ("siGab1") or scramble control siRNA ("siC"), were shown. Proliferation of these cells was also tested ( $\mathrm{H}$ and I). Gab1 expression and AktErk phosphorylations were quantified (B and G). ${ }^{*} \mathrm{p}<0.05$ vs. "shC" or "siC" group. Experiments in this Fig. were repeated three times, with similar results obtained. 
we constructed a miR-29a expression vector ("pSuper-puro-miR-29a", named as "Vec-miR$29 a$ "). Using this vector, we established stable A172 cells constitutively-expressing "Vec-miR29a". A qRT-PCR assay (Fig. 3A) confirmed that miR-29a-3p levels were significantly elevated in the stable cells. Significantly, forced-expression of miR-29a-3p depleted Gab1 (mRNA and protein) in A172 cells (Fig. 3B and C). Activation of Akt (Fig. 3C) was also inhibited in "Vec-miR-29a"-expressing cells. More importantly, A172 cell proliferation, tested using cell counting (Fig. 3D) and BrdU ELISA (Fig. 3E) assays, was suppressed with forced-expression of miR-29a-3p. Expression of microRNA-control ("miR-C") had no effect on miR-29a-3p or Gab1 expression, as well as cell proliferation (Fig. 3A-E).

Exogenous expression of the anti-sense miR-29a ("antagomiR-29a") caused further miR-29a-3p downregulation (Fig. 3F), resulting in increased Gab1 expression and Akt activation (Fig. 3G), and enhanced cell proliferation (Fig. 3H). In the primary human glioma cells, forced-expression of "Vec-miR-29a" similarly induced miR-29a-3p upregulation (Fig. 3I), Gab1 silence (Fig. 3J), Akt suppression (Fig. 3J) and cell proliferation inhibition (Fig. 3K). These studies demonstrate that forced-expression of miR-29a-3p can silence Gab1 to inhibit glioma cell proliferation.

\section{Gab1 is the primary target of miR-29a-3p in glioma cells}

As recent studies have shown that miR-29a-3p could possibly have other targets [44, 45], we next examined if Gab1 is the direct and the primary target of miR-29a-3p in human glioma cells. Luciferase activity assay results demonstrate that Gab1 mRNA 3-UTR luciferase activity was decreased significantly with forced-expression of miR-29a-3p, but was increased after antagomiR-29a expression (Fig. 4A). These results suggest that Gab1 is likely the direct target of miR-29a-3p.

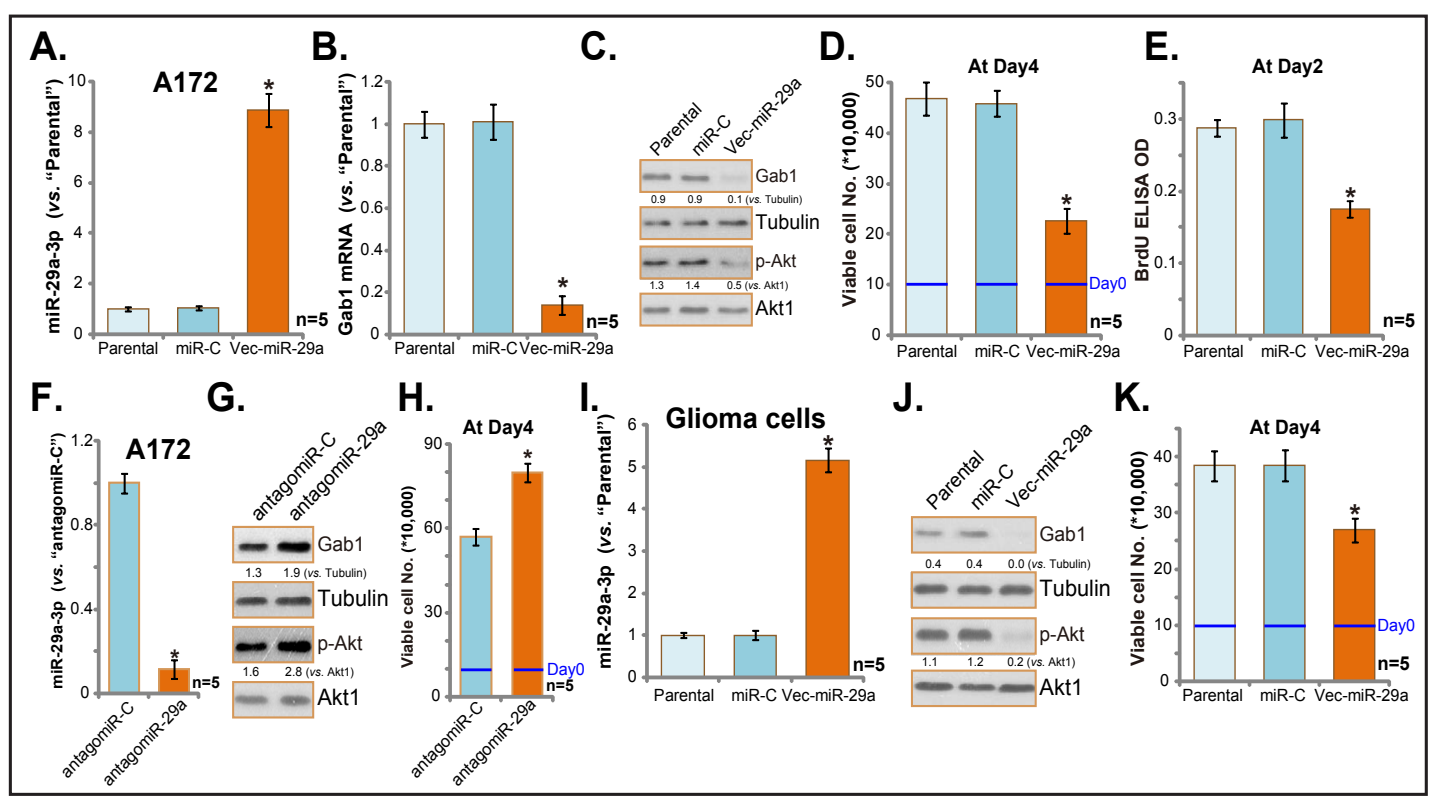

Fig. 3. Forced-expression of miR-29a-3p silences Gab1, inhibiting glioma cell proliferation. A172 glioma cells, expressing non-sense control miRNA ("miR-C"), miR-29a-pSuper-puro ("Vec-miR-29a"), as well as the anti-sense miR-29a ("antagomiR-29a") or antagomiR-control ("antagomiR-C"), were subjected to qRTPCR assay to test expression of miR-29a-3p (A and F) and Gab1 mRNA (B); Listed proteins were tested by Western blotting assay ( $C$ and $G$ ); Cell proliferation was also tested (D, E, H). The primary human glioma cells, infected with "miR-C" or "Vec-miR-29a", were also subjected to above assays to test miR-29a-3p expression (I), listed protein expression (J) and cell proliferation (K). "Parental" stands for the parental control cells. Gab1 expression and Akt-Erk phosphorylations were quantified (C, G and J). * p<0.05 vs. "miR-C" or "antagomiR-C" group. Experiments in this Fig. were repeated three times, with similar results obtained. 
If Gab1 is the primary target of miR-29a-3p, then forced-expression of miR-29a$3 p$ should not affect cells with depleted Gab1. To test this hypothesis, we exogenously expressed "Vec-miR-29a" (see Fig. 3) in Gab1-shRNAexpressing A172 cells. As demonstrated, miR-29a-3p expression (Fig. 4C) failed to further inhibit proliferation of A172 cells with Gab1 shRNAs (-a/-b) (See Fig. 2). Thus, miR29a-3p was unable to inhibit proliferation of Gab1-silenced cells, suggesting that Gab1 is the primary target of miR-29a$3 p$ in A172 cells.

In further support of this hypothesis, we constructed a 3'-UTR-mutant Gab1 (UTRG160A), that was not targeted by miR-29a-3p(Fig. 4D). Introduction of the 3'-UTR-mutant Gab1 almost completely blocked miR-29a-3p-induced inhibition of Akt activity (Fig. 4D) and proliferation (Fig. $4 \mathrm{~F}$ and G). miR-29a-3p levels were equivalent between cells with/out the 3'-UTR-mutant Gab1 (Fig. 4E). These results suggest that Gab1 is the direct and primary target of miR$29 a-3 p$ in mediating its antiglioma cell activity.

\section{Discussion}

Simultaneous activation of multiple receptor tyrosine kinases (RTKs) in human glioma is believed to cause constitutive activation of multiple oncogenic downstream signalings, including PI3K-Akt and Erk-MAPK cascades [46, 47]. RTKs, including EGFR, VEGFR, FGFR and PDGFR, are important oncotarget proteins of glioma [47]. Thus, inhibition of one of these RTKs results in partial or no inhibition of downstream signaling and weak anti-glioma activity $[46,47]$. Remarkably, the signaling transduction from these surface RTKs to downstream cascades relies heavily on adaptor/scaffold proteins, of which, Gab1 is a key adaptor protein $[10,12,48]$. We show that shRNA/siRNA knockdown of Gab1 largely attenuated Akt and Erk-MAPK activation in human glioma cells. More importantly, glioma cell proliferation was also significantly suppressed with Gab1 knockdown. Therefore, targeting

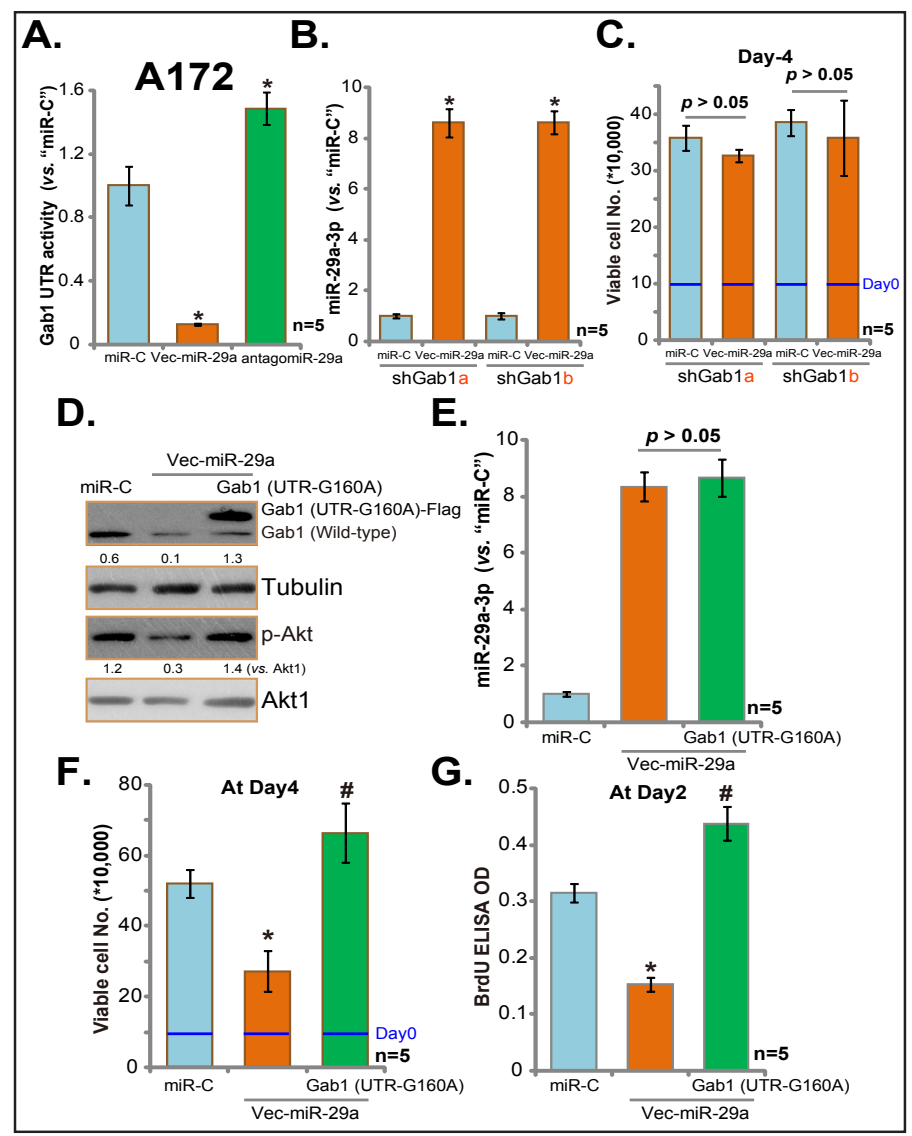

Fig. 4. Gab1 is the primary target of miR-29a-3p in glioma cells. A172 cells, expressing non-sense control miRNA ("miR-C"), miR-29a-pSuper-puro ("Vec-miR-29a") or the anti-sense miR-29a ("antagomiR29a"), were subjected to luciferase activity to test Gab1 mRNA 3'UTR activity (Fig. A). Stable A172 cells with indicated Gab1 shRNA ("-a/-b") were further constructed with "Vec-miR-29a" or "miR-C", miR-29a-3p expression (B) and cell proliferation (C) were tested. A172 cells with "miR-C" or "Vec-miR-29a" were further constructed with/out the 3'-UTR-mutant Gab1 (Gab1 UTR-G160A, tagged with Flag), listed protein expression (D), miR-29a-3p expression (E), and cell proliferation ( $F$ and G) were tested. Gab1 expression and Akt phosphorylation were quantified (D). * p<0.05 vs. "miR-C" group. * $\mathrm{p}<0.05$ vs. "Vec-miR-29a" only group. Experiments in this Fig. were repeated three times, with similar results obtained. 
the adaptor protein Gab1 should be a reasonable strategy again glioma cells, especially as its expression is significantly elevated in human glioma tissues and cells.

miR-29s are well-established anti-tumor miRNAs $[44,45]$. There are at least three members of miR-29 family miRNAs, including miR-29a, miR-29b and miR-29c [44, 45]. Aberrant expression (mainly downregulation) of miR-29 family miRNAs in multiple cancers is associated with tumorigenesis and cancer progression [44, 45]. miR-29 family miRNAs are known to target multiple tumor-related signaling proteins, including cell proliferation, cell cycle progression, cell survival, apoptosis-resistance and metastasis as well as epigenetic and immune-response [44, 45]. Therefore, miR-29s are well-established anti-tumor miRNAs $[44,45]$. We show that miR-29a is downregulated in human glioma tissues. Meanwhile, forced-expression of miR-29a induced downregulation of Gab1 and glioma cell proliferation inhibition.

In the current study, we propose that Gab1 mRNA is the target of miR-29a-3p, acting as a tumor suppressor. In both established (A172 cell line) and primary human glioma cells, forced-expression of miR-29a-3p caused Gab1 repression at both mRNA and protein level, leading to downstream Akt-Erk inhibition. Downregulation of miR-29a-3p via antagomiR29a caused Gab1 upregulation and enhanced downstream signaling activation. Significantly, the Gab1 mRNA 3-UTR activity was significantly inhibited by miR-29a-3p expression, but was enhanced with antagomiR-29a expression.

Recent studies have proposed that miR-29 could be a novel anti-cancer miR. For example, Park et al., demonstrated that miR-29 activated p53 by targeting p85alpha and CDC42, and promoted cancer cell apoptosis [49]. Furthermore, miR-29 expression led to direct downregulation of Mcl-1, an anti-apoptotic Bcl-2 family member, to promote cancer cell apoptosis [50]. In the current study, we provide evidence to support that Gab1 is the primary target protein of miR-29a-3p in mediating its anti-glioma cell activity. Forced-expression of miR-29a-3p was ineffectual against proliferation in glioma cells when Gab1 was already silenced. Remarkably, introduction of an UTR mutant Gab1 was able to completely block miR-29a-3p-induced inhibition on human glioma cells. Thus, miR-29a-3p inhibits glioma cell proliferation possibly via directly and primarily silencing its target Gab1.

The significance of miRs in diagnostic and prognostic determination has been demonstrated in glioma [20-22] and a number of other human cancers [51, 52]. In the current study, we provided the quantitative description of a potential relationship between decreased miR-29-3p expression and the grade of malignant glioma patients. According to our results, miR-29-3p is expressed at low levels in glioma, and higher WHO grade gliomas tend to have even lower expression of miR-29-3p. It will certainly be interesting to further test the significance of miR-29-3p downregulation in the diagnosis and prognosis of human glioma.

\section{Conclusion}

In summary, miR-29a-3p downregulation leads to Gab1 upregulation to promote glioma cell proliferation. Expression of miR-29a-3p to block Gab1 expression could provide a promising therapeutic strategy to inhibit RTK-mediated glioma progression.

\section{Acknowledgements}

This work was generously supported by grants from the National Natural Science Foundation of China (Nos. 81371055 and 81570859, 81302195, 31371139 and 81502162, 81571282); Grants from Natural Science Foundation of Jiangsu Province (BK20130301, BK20151213, BK20170060), and Jiangsu province Six-Talent Peaks (To C.C.). The funders had no role in study design, data collection and analysis, decision to publish, or preparation of the manuscript. All authors carried out all the experiments, participated in the design 


\section{Cellular Physiology Cell Physiol Biochem 2018;48:450-460 \begin{tabular}{l|l} 
DOI: 10.1159/000491776 & $\begin{array}{l}\text { O } 2018 \text { The Author(s). Published by S. Karger AG, Basel } \\
\text { www.karger.com/cpb }\end{array}$
\end{tabular}}

Shao et al.: microRNA-29a Targets Gab1 in Glioma Cells

of the project conceived of the study, and participated in its design and coordination and helped to draft the manuscript.

\section{Disclosure Statement}

None of the authors have any competing interests in the manuscript.

\section{References}

1 Westphal M, Lamszus K: The neurobiology of gliomas: from cell biology to the development of therapeutic approaches. Nat Rev Neurosci 2011;12:495-508.

2 Siegel R, Naishadham D, Jemal A: Cancer statistics, 2012. CA Cancer J Clin 2012;62:10-29.

-3 Siegel R, Ma J, Zou Z, Jemal A: Cancer statistics, 2014. CA Cancer J Clin 2014;64:9-29.

$\rightarrow 4$ Khasraw M, Lassman AB: Neuro-oncology: late neurocognitive decline after radiotherapy for low-grade glioma. Nat Rev Neurol 2009;5:646-647.

5 Pollack IF: Neuro-oncology: Therapeutic benefits of reirradiation for recurrent brain tumors. Nat Rev Neurol 2010;6:533-535.

6 Wang Y, Jiang T: Understanding high grade glioma: molecular mechanism, therapy and comprehensive management. Cancer Lett 2013;331:139-146.

7 Huang TT, Sarkaria SM, Cloughesy TF, Mischel PS: Targeted therapy for malignant glioma patients: lessons learned and the road ahead. Neurotherapeutics 2009;6:500-512.

8 Lefranc F, Rynkowski M, DeWitte 0, Kiss R: Present and potential future adjuvant issues in high-grade astrocytic glioma treatment. Adv Tech Stand Neurosurg 2009;34:3-35.

-9 Li X, Wu C, Chen N, Gu H, Yen A, Cao L, Wang E, Wang L: PI3K/Akt/mTOR signaling pathway and targeted therapy for glioblastoma. Oncotarget 2016;7:33440-33450.

10 Yart A, Mayeux P, Raynal P: Gab1, SHP-2 and other novel regulators of Ras: targets for anticancer drug discovery? Curr Cancer Drug Targets 2003;3:177-192.

-11 Sarmay G, Angyal A, Kertesz A, Maus M, Medgyesi D: The multiple function of Grb2 associated binder (Gab) adaptor/scaffolding protein in immune cell signaling. Immunol Lett 2006;104:76-82.

12 Liu Y, Rohrschneider LR: The gift of Gab. FEBS Lett 2002;515:1-7.

13 Gu H, Neel BG: The "Gab" in signal transduction. Trends Cell Biol 2003;13:122-130.

14 Zhang YM, Zhang ZQ, Liu YY, Zhou X, Shi XH, Jiang Q, Fan DL, Cao C: Requirement of Galphai1/3-Gab1 signaling complex for keratinocyte growth factor-induced PI3K-AKT-mTORC1 activation. J Invest Dermatol 2015;135:181-191.

15 Shioyama W, Nakaoka Y, Higuchi K, Minami T, Taniyama Y, Nishida K, Kidoya H, Sonobe T, Naito H, Arita Y, Hashimoto T, Kuroda T, Fujio Y, Shirai M, Takakura N, Morishita R, Yamauchi-Takihara K, Kodama T, Hirano T, Mochizuki N, Komuro I: Docking protein Gab1 is an essential component of postnatal angiogenesis after ischemia via HGF/c-met signaling. Circ Res 2011;108:664-675.

16 Meng S, Chen Z, Munoz-Antonia T, Wu J: Participation of both Gab1 and Gab2 in the activation of the ERK/ MAPK pathway by epidermal growth factor. Biochem J 2005;391:143-151.

17 Liu H, Li G, Zeng W, Zhang P, Fan F, Tu Y, Zhang Y: Combined detection of Gab1 and Gab2 expression predicts clinical outcome of patients with glioma. Med Oncol 2014;31:77.

$>18$ Du H, Guo L, Fang F, Chen D, Sosunov AA, McKhann GM, Yan Y, Wang C, Zhang H, Molkentin JD, Gunn-Moore FJ, Vonsattel JP, Arancio O, Chen JX, Yan SD: Cyclophilin D deficiency attenuates mitochondrial and neuronal perturbation and ameliorates learning and memory in Alzheimer's disease. Nat Med 2008;14:1097-1105.

19 Huang AL, Ostrowski MC, Berard D, Hager GL: Glucocorticoid regulation of the Ha-MuSV p21 gene conferred by sequences from mouse mammary tumor virus. Cell 1981;27:245-255.

20 Hu CE, Du PZ, Zhang HD, Huang GJ: Long Noncoding RNA CRNDE Promotes Proliferation of Gastric Cancer Cells by Targeting miR-145. Cell Physiol Biochem 2017;42:13-21.

21 Liu T, Wu C, Weng G, Zhao Z, He X, Fu C, Sui Z, Huang SX: Bufalin Inhibits Cellular Proliferation and Cancer Stem Cell-Like Phenotypes via Upregulation of MiR-203 in Glioma. Cell Physiol Biochem 2017;44:671-681. 


\section{Cellular Physiology Cell Physiol Biochem 2018;48:450-460 \begin{tabular}{l|l} 
DOI: 10.1159/000491776 & Ond Biochemistry \\
Published online: July 17, 2018 & $\begin{array}{l}\text { 2018 The Author(s). Published by S. Karger AG, Basel } \\
\text { www.karger.com/cpb }\end{array}$
\end{tabular}}

Shao et al.: microRNA-29a Targets Gab1 in Glioma Cells

22 Zhang BL, Dong FL, Guo TW, Gu XH, Huang LY, Gao DS: MiRNAs Mediate GDNF-Induced Proliferation and Migration of Glioma Cells. Cell Physiol Biochem 2017;44:1923-1938.

23 Cao C, Huang X, Han Y, Wan Y, Birnbaumer L, Feng GS, Marshall J, Jiang M, Chu WM: Galpha(i1) and Galpha(i3) are required for epidermal growth factor-mediated activation of the Akt-mTORC1 pathway. Sci Signal 2009;2:ra17.

24 Cao C, Rioult-Pedotti MS, Migani P, Yu CJ, Tiwari R, Parang K, Spaller MR, Goebel DJ, Marshall J: Impairment of TrkB-PSD-95 signaling in Angelman syndrome. PLoS Biol 2013;11:e1001478.

-25 Yang L, Zheng LY, Tian Y, Zhang ZQ, Dong WL, Wang XF, Zhang XY, Cao C: C6 ceramide dramatically enhances docetaxel-induced growth inhibition and apoptosis in cultured breast cancer cells: a mechanism study. Exp Cell Res 2015;332:47-59.

-26 Li ZW, Cai S, Liu Y, Yang CL, Tian Y, Chen G, Cao C: Over-expression of Galphai3 in human glioma is required for Akt-mTOR activation and cell growth. Oncotarget 2016;10.18632/oncotarget.10995

27 Cai S, Li Y, Bai JY, Zhang ZQ, Wang Y, Qiao YB, Zhou XZ, Yang B, Tian Y, Cao C: Galphai3 nuclear translocation causes irradiation resistance in human glioma cells. Oncotarget 2017;8:35061-35068.

28 Liu YY, Chen MB, Cheng L, Zhang ZQ, Yu ZQ, Jiang Q Chen G, Cao C: microRNA-200a downregulation in human glioma leads to Galphai1 over-expression, Akt activation, and cell proliferation. Oncogene 2018;10.1038/s41388-018-0184-5

29 Gong YQ, Huang W, Li KR, Liu YY, Cao GF, Cao C, Jiang Q: SC79 protects retinal pigment epithelium cells from UV radiation via activating Akt-Nrf2 signaling. Oncotarget 2016;10.18632/oncotarget.11164]

-30 Li KR, Yang SQ, Gong YQ Yang H, Li XM, Zhao YX, Yao J, Jiang Q, Cao C: 3H-1, 2-dithiole-3-thione protects retinal pigment epithelium cells against Ultra-violet radiation via activation of Akt-mTORC1-dependent Nrf2-HO-1 signaling. Sci Rep 2016;6:25525.

-31 Zhang H, Liu YY, Jiang Q, Li KR, Zhao YX, Cao C, Yao J: Salvianolic acid A protects RPE cells against oxidative stress through activation of Nrf2/HO-1 signaling. Free Radic Biol Med 2014;69:219-228.

-32 Tang CZ, Li KR, Yu Q, Jiang Q Yao J, Cao C: Activation of Nrf2 by Ginsenoside Rh3 protects retinal pigment epithelium cells and retinal ganglion cells from UV. Free Radic Biol Med 2018;117:238-246.

-33 Schmittgen TD, Zakrajsek BA: Effect of experimental treatment on housekeeping gene expression: validation by real-time, quantitative RT-PCR. J Biochem Biophys Methods 2000;46:69-81.

-34 Seiden-Long I, Navab R, Shih W, Li M, Chow J, Zhu CQ, Radulovich N, Saucier C, Tsao MS: Gab1 but not Grb2 mediates tumor progression in Met overexpressing colorectal cancer cells. Carcinogenesis 2008;29:647655.

35 Zhang J, Song Y, Zhang C, Zhi X, Fu H, Ma Y, Chen Y, Pan F, Wang K, Ni J, Jin W, He X, Su H, Cui D: Circulating MiR-16-5p and MiR-19b-3p as Two Novel Potential Biomarkers to Indicate Progression of Gastric Cancer. Theranostics 2015;5:733-745.

-36 Yang J, Zhang Z, Chen C, Liu Y, Si Q, Chuang TH, Li N, Gomez-Cabrero A, Reisfeld RA, Xiang R, Luo Y: MicroRNA-19a-3p inhibits breast cancer progression and metastasis by inducing macrophage polarization through downregulated expression of Fra-1 proto-oncogene. Oncogene 2014;33:3014-3023.

-37 Heimberg AM, Cowper-Sal-lari R, Semon M, Donoghue PC, Peterson KJ: microRNAs reveal the interrelationships of hagfish, lampreys, and gnathostomes and the nature of the ancestral vertebrate. Proc Natl Acad Sci U S A 2010;107:19379-19383.

38 Chen B, She S, Li D, Liu Z, Yang X, Zeng Z, Liu F: Role of miR-19a targeting TNF-alpha in mediating ulcerative colitis. Scand J Gastroenterol 2013;48:815-824.

-39 Li X, Wang D, Chen Z, Lu E, Wang Z, Duan J, Tian W, Wang Y, You L, Zou Y, Cheng Y, Zhu Q, Wan X, Xi T, Birnbaumer L, Yang Y: Galphai1 and Galphai3 regulate macrophage polarization by forming a complex containing CD14 and Gab1. Proc Natl Acad Sci U S A 2015;112:4731-4736.

40 Liu M, Wang Z, Yang S, Zhang W, He S, Hu C, Zhu H, Quan L, Bai J, Xu N: TNF-alpha is a novel target of miR19a. Int J Oncol 2011;38:1013-1022.

41 Zhang XP, Li KR, Yu Q Yao MD, Ge HM, Li XM, Jiang Q, Yao J, Cao C: Ginsenoside Rh2 inhibits vascular endothelial growth factor-induced corneal neovascularization. FASEB J 2018;10.1096/ fj.201701074RRfj201701074RR.

-42 Zheng B, Mao JH, Li XQ, Qian L, Zhu H, Gu DH, Pan XD: Over-expression of DNA-PKcs in renal cell carcinoma regulates mTORC2 activation, HIF-2alpha expression and cell proliferation. Sci Rep 2016;6:29415. 


\section{Cellular Physiology Cell Physiol Biochem 2018;48:450-460 and Biochemistry Published on/:00491776, $2018 \quad \begin{aligned} & \text { DO 2018 The Author(s). Published by S. Karger AG, Basel } \\ & \text { www.karger.com/cpb }\end{aligned}$ \\ Shao et al.: microRNA-29a Targets Gab1 in Glioma Cells}

43 Marshall J, Zhou XZ, Chen G, Yang SQ, Li Y, Wang Y, Zhang ZQ, Jiang Q, Birnbaumer L, Cao C: Antidepression action of BDNF requires and is mimicked by Galphai1/3 expression in the hippocampus. Proc Natl Acad Sci U S A 2018;115:E3549-E3558.

44 Schmitt MJ, Margue C, Behrmann I, Kreis S: MiRNA-29: a microRNA family with tumor-suppressing and immune-modulating properties. Curr Mol Med 2013;13:572-585.

45 Jiang H, Zhang G, Wu JH, Jiang CP: Diverse roles of miR-29 in cancer (review). Oncol Rep 2014;31:15091516.

-46 Lo HW: EGFR-targeted therapy in malignant glioma: novel aspects and mechanisms of drug resistance. Curr Mol Pharmacol 2010;3:37-52.

47 Snuderl M, Fazlollahi L, Le LP, Nitta M, Zhelyazkova BH, Davidson CJ, Akhavanfard S, Cahill DP, Aldape KD, Betensky RA, Louis DN, Iafrate AJ: Mosaic amplification of multiple receptor tyrosine kinase genes in glioblastoma. Cancer Cell 2011;20:810-817.

-48 Eswarakumar VP, Lax I, Schlessinger J: Cellular signaling by fibroblast growth factor receptors. Cytokine Growth Factor Rev 2005;16:139-149.

49 Park SY, Lee JH, Ha M, Nam JW, Kim VN: miR-29 miRNAs activate p53 by targeting p85 alpha and CDC42. Nat Struct Mol Biol 2009;16:23-29.

50 Mott JL, Kobayashi S, Bronk SF, Gores GJ: mir-29 regulates Mcl-1 protein expression and apoptosis. Oncogene 2007;26:6133-6140.

51 Calin GA, Croce CM: MicroRNA signatures in human cancers. Nat Rev Cancer 2006;6:857-866.

52 Orang AV, Barzegari A: MicroRNAs in colorectal cancer: from diagnosis to targeted therapy. Asian Pac J Cancer Prev 2014;15:6989-6999. 